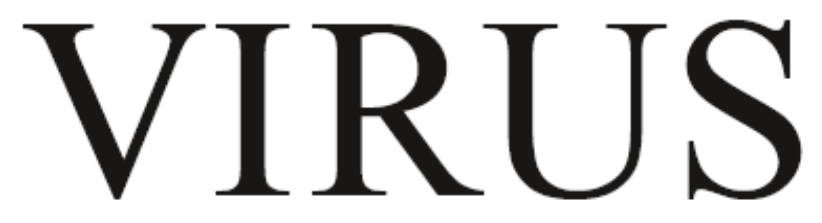

Beiträge zur Sozialgeschichte der Medizin

\title{
Band 9
}

Herausgegeben von

Carlos Watzka, Elisabeth Dietrich-Daum und Andreas Golob

für den Verein für Sozialgeschichte der Medizin

Wien: Verlagshaus der Ärzte, 2010

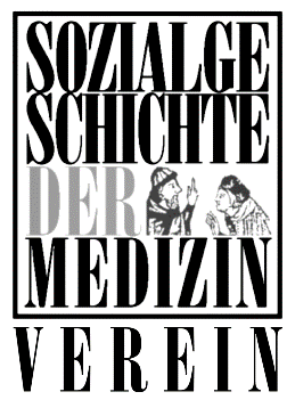




\title{
Das staatliche „Fürsorgewesen“ in der Bezirkshauptstadt Leoben von den Anfängen um 1900 bis 1938
}

\author{
Eine Übersicht auf Grundlage der regionalen Presseberichterstattung
}

Die vielfältigen Aufgaben im Bereich der sozialen Wohlfahrt waren vor der Errichtung der ersten staatlichen Fürsorgestellen vielfach von engagierten Badern, Ärzten und Hebammen privat und auf eigene Kosten übernommen worden. Auch kirchliche Einrichtungen trugen einen erheblichen Anteil dazu bei, dass Bedürftigen geholfen wurde. Der größte Teil der Tätigkeiten aber, der ab dem Jahr 1896 von den ersten in der Steiermark errichteten Fürsorgestellen übernommen wurde, war in den vorausgegangenen Jahrhunderten von „Armenärzten“ ausgeführt worden. Diese waren mit Geldern der „öffentlichen Hand“ dafür - meist kärglich -entlohnt worden. Auch in Leoben, der zweitgrößten Stadt der Steiermark, wurden eigene Armenärzte vom Magistrat beziehungsweise von der Gemeinde für ihre Tätigkeit honoriert. Dafür hatten sie bedürftige Kranke unentgeltlich zu behandeln. ${ }^{1}$

In der zweiten Hälfte des 19. Jahrhunderts war mit der im Eiltempo voranschreitenden Industrialisierung die Bevölkerung in Leoben innerhalb weniger Jahrzehnte von etwa 3.000 Einwohnern auf rund 26.000 Menschen angewachsen und damit hatten sich auch die sozialen Probleme potenziert. Mit großer Wohnungsnot, ständigem Wassermangel, Problemen bei der Abwasserbeseitigung, Missständen bei der Lebensmittelversorgung und auch der hohen Kindersterblichkeit sollen nur einige der Hauptprobleme angedeutet werden. Hiervon waren vor allem die Menschen im Bereich Donawitz, wo die Schwerindustrie angesiedelt war, und jene in der Vorstadt Mühltal, mit der Durchzugstraße von Bruck in das obere Murtal, betroffen. ${ }^{2}$

Die hohe Kindersterblichkeit und die rückläufigen Geburtenzahlen ließen zu Beginn des 20. Jahrhunderts so manchen, nicht nur in Leoben, um das Fortbestehen des - deutschen - Volkes fürchten. In Deutschland wurden aus diesem Grund bereits im Jahr 1905 erste Fürsorgestellen mit staatlich geschulten Fürsorgeschwestern eingerichtet. In Wien und anderen größeren Städten Österreichs entstanden kurz danach ähnliche Fürsorgestellen. ${ }^{3}$ Eine auffällig hohe Kindersterblichkeit vor allem in den Bal-

1 Obersteirische Volkszeitung, Nr. 142, 22.12.1921, S. 2; Nr. 104, 28.9.1922, S. 2, Nr. 113, 19.10.1922, S.2.

2 Elfriede HUBER-REISMANN, Die Medizinische Versorgung der Stadt Leoben vom 13. bis zum 20. Jahrhundert. Eine sozialhistorische Quellenstudie als Beitrag zur Medizingeschichte sowie zur Steirischen Stadtgeschichtsforschung. (Graz 2009) 
lungszentren rund um die großen Industriebetriebe war der spezifische Anlass, sich um eine bessere Beratung der Mütter in Bezug auf Säuglingspflege zu bemühen.

Eine erste Möglichkeit, Zugang auch zu den Leobner Frauen und Müttern zu finden, war eine Wiener Wanderausstellung über Säuglingsfürsorge, die im Oktober 1916, mitten im Ersten Weltkrieg, in der Stadt gezeigt wurde. Die Eröffnung der Ausstellung erfolgte am 4. Oktober unter Beisein zahlreicher städtischer Honoratioren. Anschaulich sollte der richtige Umgang mit dem Säugling erläutert sowie diesbezügliche Ernährungsund Gesundheitsfragen beantwortet und mit überlieferten falschen Vorstellungen aufgeräumt werden. In drei ausführlichen Artikeln wurde diese kostenlose, aber lehrreiche Ausstellung, die zehn Tage lang im Turnsaal der Leobner Volksschule zu sehen war, in der Obersteirischen Volkszeitung beworben. ${ }^{4}$

Als nach den Ersten Weltkrieg die Not vor allem unter den Arbeiterfamilien in Leoben besonders groß war, viele Kinder an Unterernährung litten und nicht wenige Säuglinge aus Unwissenheit falsch behandelt wurden, setzte man sich von Seiten der Leobner Ärzteschaft, des Verwaltungsbezirkes sowie der Gemeinde für eine Verbesserung der Situation ein und begründete 1919 die erste Fürsorgestelle. Unterstützt wurde die Fürsorge in der Steiermark bald auch vom amerikanischen Roten Kreuz, das sich um den Aufbau dieser Einrichtung verdient machte. Zum Fürsorgebereich gehörte damals vor allem die Betreuung der Schwangeren, der jungen Mütter, der Säuglinge und der Kleinkinder sowie der Tuberkulosekranken.

Das im Wesentlichen nach dem Ersten Weltkrieg aufgebaute Fürsorgewesen in Leoben umfasste, wie der Obersteirischen Volkszeitung zu entnehmen ist, zwei Beratungsstellen: In Donawitz wurden Mütter, die in Göss, Leitendorf, Hinterberg und Donawitz wohnten, zwei Mal pro Monat im Kinderheim in der Buchmüllerstraße beraten. Getrennt davon wurde in der Vordernbergerstraße eine Säuglings- und Mütterberatungsstelle ausschließlich für die Werksangehörigen der ÖAMG eingerichtet, die von Werksarzt Dr. Gustav Hackl betreut wurde.

Frauen aus Judendorf, Nennersdorf und Leoben fanden in einer zweiten Bezirksberatungsstelle Hilfe und Beratung. Diese wurde im Jahr 1919 zunächst jeden zweiten und vierten Dienstagvormittag in einem Raum der Leobner Bezirksvertretung angeboten, bis die neue Lokalität in der Kaserne in der Peter-Tunner-Straße fertig gestellt werden konnte. Die Mütter wurden damals ausdrücklich aufgefordert, ihre Kinder mitzubringen. Der Saal sei geheizt und die Kinder könnten abgewogen werden.

Die beiden Beratungsstellen erlebten nach der Gründung eines übergeordneten „Zweigvereins zur Förderung der Volksgesundheit“ bald einen deutlichen Aufschwung. Anfänglich, im Jahr 1919, mussten die Frauen aber mit zahlreichen Vergünstigungen regelrecht in die Fürsorgestelle „gelockt" werden. So gab es beispielsweise für Schwangere besondere Karten, die ihnen den Vortritt in der leidigen Warteschlange ermöglichten. Mütter bekamen für ihre Säuglinge Gries, Weiß- oder Nährmehle ,Milch und Zucker - die sonst kaum in ausreichender Menge auf den Lebensmittelkarten zu finden waren - sowie günstige Windeln und Kinderkleidung, worauf in der lokalen Presse ausdrücklich hingewiesen wurde. ${ }^{6}$

4 Obersteirische Volkszeitung, Nr. 79, 4.10.1916, S. 4, Nr. 80, 7.10.1916, S. 4, Nr. 81, 11.10.1916, S. 4.

5 Obersteirische Volkszeitung, Nr. 89, 8.11.1919, S. 3; Nr. 95, 29.11.1919, S. 4. Im Jahr 1934 übersiedelte die Bezirksfürsorgestelle in Leoben in neue Räumlichkeiten. Vgl. Obersteirische Volkszeitung, Nr. 112, 15.9.1934, S. 4. 
Der genannte Verein bemühte sich zu Beginn der 1920er Jahre dann auch um die Verteilung von Spenden für bedürftige Kleinkinder. Im Jahr 1921 waren Lebensmittelspenden aus England zur Verteilung gelangt. Im Jahr darauf hatte das amerikanische Rote Kreuz eine größere Menge an Babywäsche und Kleidung für Kinder bis etwa zwei Jahren zur Verfügung gestellt, die zu sehr günstigen Preisen verkauft wurde. Diese durfte, das war als Bedingung festgelegt worden, nur an jene Kinder verteilt werden, die regelmäßig von der Fürsorgestelle betreut wurden. Zehn Prozent der Hilfesuchenden in Leoben wurden aufgrund ihrer Bedürftigkeit mit Wäsche beschenkt.

Als sich das amerikanische Rote Kreuz aus der Fürsorge zurückzog, musste die Finanzierung der Einrichtungen Ende 1922 neu geregelt werden. Betroffen waren die Gemeinden Leoben, Donawitz, Göss, Proleb, Niklasdorf und St. Peter Freienstein. Staatliche Vorschläge, ein Komitee zu gründen, dem die Aufbringung der nötigen finanziellen Mittel überlassen werden sollte, schienen zu schwerfällig. Nach längeren Diskussionen einigten sich die besagten Gemeinden im so genannten erweiterten Fürsorgeausschuss darauf, dass der Verein zur Förderung der Gesundheitspflege weiterhin die Fürsorge vor Ort durchführen sollten, die Gemeinden aber die Kosten für das Personal, die Räumlichkeiten, die Beheizung und die Beleuchtung tragen würden. Ein Drittel der Personalkosten wurde von Seiten des Staates zugeschossen. Vom Verein wurde nur die Kranken- und Pensionsversicherung für das Personal bezahlt. In diesem Rahmen war neben der Säuglings- und Mütterberatung auch die Tuberkulosenfürsorge inbegriffen. ${ }^{7}$

Mit Dezember 1922 errichtete die Alpine-Montangesellschaft für ihre Bergwerksangehörigen eine eigene Fürsorgestelle und stellte eine Fürsorgerin ein, die nun ausschließlich für die Arbeiterfamilien in den Bergwerken Seegraben und Münzenberg zuständig war. Durch diese Maßnahme verringerte sich die Zahl der Ratsuchenden in den anderen beiden Beratungsstellen um einige hundert.

Als Fürsorgearzt in den Bezirksfürsorgestellen war ab 1922 der damalige Bezirksarzt Dr. Alfred Kraemer bestellt. Als Fürsorgerin war Elise Jeschek und als Hilfsfürsorgerin Antonia Michlmayr beschäftigt. Ab Oktober 1922 wurde das Angebot erweitert und nun fanden Dienstags und Freitags von 9 bis 12 Uhr ärztliche Sprechstunden statt. Die Statistik für das Jahr 1922 wies 1.501 „befürsorgte“ Personen aus, ${ }^{8}$ darunter 570 Säuglinge, 345 Kinder von 1 bis 2 Jahren und 22 Kinder von 2 bis 3 Jahren, aber auch 160 Schwangere und 404 stillende Mütter. Drei Viertel der betreuten Kinder waren ehelich geboren, und 70 Prozent der betreffenden Kinder wurden von ihren Müttern gestillt. Elf betreute Kinder starben im Jahr 1922, wobei sich die Sterblichkeit von gestillten und künstlich ernährten Kindern beinahe die Waage hielt. Als häufigste Todesursache, sieben Kinder waren davon betroffen, wurde Lungenentzündung angegeben. Sieben Kinder starben noch bevor sie das erste Lebensjahr vollendet hatten. Die beiden Fürsorgerinnen hatten in diesem Jahr 1.828 Hausbesuche absolviert. Den „Befürsorgten“ wurden 3.887 Kilogramm Gries und 2.217 Kilogramm „Nestlemehl“ sowie Seife zu günstigsten Preisen verkauft, dazu kam noch billige Kinderkleidung für 632 Befürsorgte. Allein diese Zahlen aus dem Jahr 1922 zeigen die dringende Notwendigkeit der Fürsorgestellen in Leoben sowie deren großartige Leistung. ${ }^{9}$

\footnotetext{
7 Obersteirische Volkszeitung, Nr. 48, 24.4.1923, S. 2.

8 Obersteirische Volkszeitung, Nr. 48, 24.4.1923, S. 2.

9 Obersteirische Volkszeitung, Nr. 48, 24.4.1923, S. 2.
} 
Der publizierte Fürsorgebericht für das Jahr 1923 wies ähnliche Zahlen auf. Schwangere kamen aber kaum noch in die Fürsorgestelle, da für sie die Schwangerenzubußen weggefallen waren und nun kaum noch Anreiz bestand, diese Fürsorgestelle aufzusuchen. ${ }^{10}$ Im Jahr 1924 schied die Gemeinde Proleb aus dem Fürsorgeausschuss aus und organisierte die Gemeindefürsorge selbständig. Im Frühjahr des darauf folgenden Jahres beschloss auch die Gemeinde Leoben, sich aus dem Fürsorgeausschuss zurückzuziehen. Diese Entscheidung fiel im Gemeinderat mit Mehrheitsbeschluss. Auslöser dafür war der Entschluss des Fürsorgeausschusses, einen bestimmten Beamten zur gemeinsamen Verwaltung der Fürsorge einzustellen, dessen Auswahl die heftigsten Reaktionen bei den bürgerlichen Mehrheitsparteien im Leobner Gemeinderat auslöste. Dieser Kantonismus konnte auf Dauer jedoch nicht gut gehen. Ende 1925 plante die Bezirksvertretung, die Bezirksfürsorge auf den gesamten Bezirk Leoben auszudehnen und dabei alle Gemeinden mit einzubeziehen. Die entsprechende Sitzung fand am 9. Dezember 1925 statt, wobei der Bezirksobmann bekannt gab, dass hinkünftig die Bezirksfürsorge aufgrund der Vereinfachung der Leitung und Durchführung ohne Einfluss der Gemeinden stattfinden sollte. Dieses Vorhaben störte die Gemeinderäte der Deutschen Gemeindepartei ebenso wie die der Nationalistischen Partei. Der Leobner städtische Fürsorgeausschuss beschloss daraufhin, sich nicht dem Bezirk anzuschließen und die Fürsorge nur im eigenen Wirkungskreise durchzuführen. Die Minderheitsfraktion der Sozialdemokraten im Gemeinderat war dagegen für einen Zusammenschluss. ${ }^{11}$

Strittig waren vor allem die wahren Kosten, die auf die Gemeinde zukommen würden. Bislang hatte die Gemeinde etwa 23.000 Schilling pro Jahr für sämtliche Fürsorgeausgaben bereitstellen müssen. Der nun errechnete Betrag von nur rund 7.600 Schilling schien unrealistisch, da bei der Berechnung davon ausgegangen worden war, dass sämtliche Gemeinden des Bezirkes an diesem Projekt teilnehmen würden, wobei zu dieser Zeit aufgrund verschiedener Gemeinderatsbeschlüsse bereits feststand, dass sich nur die sozialdemokratischen Gemeinden beteiligen wollten. Die Projektgegner gingen davon aus, dass realistischer Weise von der Gemeinde Leoben mehr als 12.000 Schilling jährlich zu bezahlen wären. Ein weiterer Grund für die ablehnende Haltung war, dass die Mehrheit der teilnehmenden Gemeinden sozialdemokratisch regiert war und so im Bezirksfürsorgeausschuss mehrheitlich Sozialdemokraten vertreten wären. Die anderen Parteien sahen dadurch ein Ungleichgewicht in der Mitbestimmung, vor allem dann, wenn es sich um strittige Fragen wie Personalentscheidungen handeln werde. In der Vergangenheit hatte sich dies bereits einige Male gezeigt. Da außer dem Obmann bisher nur Sozialdemokraten diesem Ausschuss angehört hatten, befürchtete man, dass die eher bürgerliche Gemeinde Leoben nicht ausreichend gut vertreten sein würde. Genährt wurden diese Befürchtungen der anderen im Gemeinderat vertretenen Parteien auch dadurch, dass bei den Vorverhandlungen ausschließlich Sozialdemokraten über das zukünftige Fürsorgewesen diskutierten und weder ein Fachmann - hier wies man auf den langjährigen Fürsorgearzt Dr. Alfred Kraemer hin - noch Nicht-Sozialdemokraten um ihre Meinung gefragt worden seien. Schließlich sollte über den Beschluss, sich dem Bezirksfürsorgeprojekt nicht anzuschließen, Ende Dezember 1925 im Leobner Gemeinderat abgestimmt werden. Diese Abstimmung kam aber nicht zustande, da die Sozialde-

10 Obersteirische Volkszeitung, Nr. 28, 8.3.1924, S. 7.

11 Obersteirische Volkszeitung, Nr. 144, 22.12.1925, S. 2. 
mokraten durch geschlossenes Verlassen der Gemeinderatsitzung dies verhinderten. ${ }^{12}$ Nach zähem Ringen schloss sich die Stadtgemeinde Leoben dann schließlich doch der gemeinsamen Bezirksfürsorge an.

Die Mütterberatungs- und Säuglingsfürsorgestelle in Leoben wurde 1926 von durchschnittlich 25 Müttern pro Vormittag aufgesucht. Die Kinder wurden zuerst gewogen und gemessen und anschließend vom Fürsorgearzt Dr. Kraemer genau untersucht. Eine Tafel mit der Aufschrift „Fragen werden stets gerne beantwortet" sollte den Müttern die Angst vor dem Fragen nehmen. Ein Zeitungsbericht vom 25. März 1926 schildert ausführlich einen Vormittag in der Fürsorgestelle. Aus diesem Bericht lässt sich auch entnehmen, dass jene Mütter, die von sich aus mit ihren Kindern zur Fürsorgestelle kamen, sich an einer Beratung auch sehr interessiert zeigten und trotz oft schlechtester sozialer Verhältnisse ihr Bestmöglichstes für ihre Kinder taten. Die „echten“ Problemfälle erschienen hingegen meist gar nicht erst vor dem Fürsorgearzt. ${ }^{13}$

Mit 1. April 1926 ermöglichte auch die Bezirkskrankenkasse Leoben eine Fürsorgeberatung speziell für ihre Mitglieder. Die Beratung in der Leobner Bezirksfürsorgestelle war im Gegensatz dazu, an keine Kassenzugehörigkeit gebunden. Dr. Karl Ottenthaler, Dr. Franz Pechan und Dr. Hans Weiß waren als Fürsorgeärzte ausgewählt und ihnen die potentiellen Leobner Ratsuchenden nach Straßenzügen zugeteilt worden. In Göss konnten die interessierten Frauen zwischen Dr. Josef Mandl und Dr. Paul Schweighofer wählen. ${ }^{14}$

Im Jahr 1929 erhielt in Donawitz die Werksfürsorgestelle neue Räumlichkeiten in der Lorberaustraße, welche am 20. April eröffnet wurden. Sogleich wurden 86 Säuglinge und Kleinkinder „befürsorgt“. Im Dezember desselben Jahres wurde in der Bezirksvertretungssitzung dann darüber diskutiert, ob in Donawitz eine bezirkseigene Schwangerenberatung und eine Tuberkulosenfürsorgestelle eingerichtet werden sollte. Die Kosten dafür sollten sich auf etwa 12.000 Schilling jährlich belaufen. Die Gesamtausgaben des Bezirkes für das Fürsorgewesen hatten für das Jahr 1928193.107 Schilling und 55 Groschen betragen. ${ }^{15}$

Was die Inhalte der Beratungstätigkeit für Mütter von Säuglingen und Kleinkindern betrifft, so stand damals eine Reduktion der hohen Säuglingssterblichkeit ganz oben auf der Prioritätenliste. Für diese wurde von der damals noch jungen Sozialmedizin vornehmlich eine falsche Ernährung der kleinen Kinder verantwortlich gemacht; man förderte besonders das Stillen, da durch die Gabe von unbehandelter Kuhmilch häufig tödliche Magen-Darm-Erkrankungen ausgelöst wurden. Von den Krankenkassen wurden so genannte „Stillprämien“ bezahlt, und die intensive Aufklärung in Wort und Schrift trugen ebenfalls zur Stillförderung bei. Man beobachtete aufmerksam, dass diese Maßnahmen den bis dahin bestehenden „Sommergipfel“ der Kindersterblichkeit absenken halfen. Auch wurde schon Ende der 1920er Jahre durch vergleichende Analysen festgestellt, dass die Säuglingssterblichkeit in jenen Gebieten, in denen es eine gute ausgebaute Fürsorge gab, eindeutig zurückgegangen war. ${ }^{16}$

Eine eigene Schwangerenfürsorgestelle wurde in Leoben auf Anregung des Kinder-

12 Obersteirische Volkszeitung, Nr. 144, 22.12.1925, S. 2.

13 Obersteirische Volkszeitung, Nr. 36, 25.3.1926, S. 4.

14 Obersteirische Volkszeitung, Nr. 39, 1.4.1926, S. 3.

15 Obersteirische Volkszeitung, Nr. 140, 7.12.1929, S. 8.

16 Obersteirische Volkszeitung, Nr. 29, 7.3.1936, S. 4. 
arztes Dr. Karl Jellenigg eingerichtet. Gleich nach seiner Wahl in den Gemeinderat im Jahr 1924 brachte er seinen Vorschlag zum diesbezüglichen Ausbau der Fürsorgeeinrichtungen der Stadt dazu ein. Da damals die gesamten Fürsorgeeinrichtungen der Stadt dem Leobner Zweigverein des „Vereines zur Förderung der Volksgesundheit“ übertragen war, musste dieser dazu erst befragt werden. Ende Oktober 1924 konnte über Antrag dieses Vereines sodann vom erweiterten Fürsorgeausschuss der Beschluss gefasst werden, eine weitere Fürsorgestelle einzurichten. Da es damals noch keine vergleichbare Schwangerenfürsorgestelle in der Steiermark gab, musste hier Pionierarbeit geleistet und Informationen von anderswo - Wiener Neustatt hatte damals eine vorbildliche Beratungsstelle - eingeholt werden. So verzögerte sich die Ausschreibung zur Anstellung einer geeigneten „Fürsorgeschwester". ${ }^{17}$

Am 4. März 1925 konnte die Schwangerenfürsorgestelle in Räumlichkeiten des damaligen Gymnasiums eingerichtet werden, welche schon der Mütter- und Säuglingsfürsorge offen standen. Die Zuständigkeit dieser Stelle in Leoben erstreckte sich über die Gemeinden Donawitz, Göss, Leoben, Niklasdorf und St. Peter Freienstein. Die fachärztliche Leitung wurde zuerst Kinderfacharzt Dr. Karl Jellenigg und noch im Laufe des Jahres 1925 Frauenfacharzt Dr. Josef Gmeiner übertragen. Als Fürsorgeschwester wurde die diplomierte Hebamme Josefine Stoiser aufgenommen. Zur Finanzierung trugen die Bezirkskrankenkasse und die Arbeiterkranken- und Unterstützungskasse bei. Die vorerst festgesetzte eine Wochenstunde Beratungszeit wurde bald auf zwei Stunden ausgedehnt. Langsam, aber stetig nahm die Zahl der Ratsuchenden zu, und auch die Hebammen, die bisher die Betreuung der Schwangeren selbständig durchgeführt hatten, schickten von ihnen betreute, werdende Mütter nun zum Teil auch zu dieser Beratungsstelle. Die durchschnittliche Inanspruchnahme der Beratungsstelle lag bald bei etwa 10 Frauen pro Woche. Nach etwa zwei Jahren Betriebszeit versuchte man die Besucherinnenfrequenz weiter zu erhöhen und die Einrichtung richtiggehend zu bewerben:

„Der Zweck der Fürsorgestelle ist“, so berichtete die lokale Presse 1927, „fachärztliche Beratung der Frauenwelt in den der Erfüllung ihres natürlichen Berufes gewidmeten Zeitläufen und Unterstützung in der ersten Zeit des Wochenbettes. " 18

Die Fürsorge teilte sich in einen ärztlichen, einen pflegerischen und einen rechtlichen Teil. So unterstützte man vor allem die ledigen Schwangeren bei der Geltendmachung ihrer Rechte gegenüber dem „glaubhaft gemachten“ Vater, der gesetzlich verpflichtet war, für die Entbindungskosten, die Unterstützung der Mutter sechs Wochen nach der Geburt und die Versorgung des Kindes über drei Monate nach der Geburt aufzukommen. Da dieser Antrag bis zum sechsten Schwangerschaftsmonat vor Gericht eingebracht werden musste, war hier eiligste Vorsorge notwendig, um den Müttern große finanzielle Probleme und ernste Notlagen zu ersparen. Streng getrennt von der allgemeinen Fürsorge wurde in jedem Fall die medizinische Behandlung allfälliger Krankheiten. Die erkrankten Ratsuchenden wurden im Bedarfsfall an Ärzte oder Krankenanstalten weiterverwiesen. ${ }^{19}$

Besonders häufig wurde die Fürsorgestelle mit der Wohnungsnot, bevorstehender Delogierung oder Obdachlosigkeit werdender Mütter aus den unteren sozialen Schichten konfrontiert. In einigen Fällen konnten die Frauen wenigsten für die Zeit der Ent-

17 Obersteirische Volkszeitung, Nr. 25, 3.3.1925, S. 3

18 Leobner Zeitung Nr. 89, 4.8.1927, S. 1 - 2.

19 Leobner Zeitung Nr. 89, 4.8.1927, S. 1 - 2. 
bindung und des Wochenbettes an die Landesgebärklinik in Graz überwiesen werden. Bedürftige Frauen konnten sich auch für die Zuteilung von Wochenbettutensilien wie Betteinlagen und dergleichen vormerken lassen. Diese bekamen sie gleich nach der Geburt ausgehändigt. Die Fürsorgerinnen übernahmen in bestimmten Fällen für einige Tage auch die Betreuung der Kindern und des Haushalts von Frauen im Wochenbett. Die Pflege der Mütter blieb in den Händen der Hebammen. ${ }^{20}$

In den Bereich der Fürsorge fielen bald auch die Schulärzte, da an diese die Akten der Säuglings- und Kleinkinderfürsorge weitergereicht wurden. Praktische Ärzte und später auch Zahnärzte wurden aus Steuermitteln dafür bezahlt, dass sie die Schüler in den Leobner Schulen auf ihren Gesundheitszustand hin untersuchten, sodass gegebenenfalls vorbeugende oder heilende Maßnahmen gesetzt werden konnten. Schon im Jahr 1904 hatte die Bezirksvertretung Leoben vorgeschlagen, bei der Anstellung von Schulärzten darauf zu achten, das praktische Ärzte eingestellt würden, die auch über eine Ausbildung als Zahnarzt verfügten oder sich zumindest fachkundig auch mit dem Plombieren von Zähnen bei Schulkindern befassen könnten. ${ }^{21}$ Die Aufgaben der Schulärzte wuchsen in den ersten Jahrzehnten des 20. Jahrhunderts derart an, dass sich die bevölkerungsreiche Gemeinde Donawitz 1919 genötigt sah, auf Vorschlag der Sanitätssektion gemeinsam mit der Gemeinde Göss, einen eigenen Schularzt anzustellen. Dieser sollte wünschenswerter Weise ein ausgebildeter Kinderarzt sein und keine Privatpraxis betreiben. ${ }^{22}$ Der erste Facharzt für Kinderheilkunde ließ sich übrigens erst fünf Jahre nach diesem Gemeinderatsbeschluss in Leoben nieder. ${ }^{23}$

Die schulärztlichen Untersuchungen wurden gleich nach dem Ende des Ersten Weltkrieges verstärkt, da es galt, den Kampf gegen Unterernährung und mangelhafte Versorgung aufzunehmen. Manche Eltern standen aber der Einrichtung der Schulfürsorge sehr skeptisch gegenüber, fühlten sich kontrolliert und überwacht. Bei Schuleintritt wurde den Eltern ein Fragebogen mit rund 40 Fragen vorgelegt, der ausgefüllt werden musste und die Grundlage für das „Stammblatt“ bildete, das den Schüler durch die gesamte Schulzeit begleitete und dem Schularzt eine laufende Kontrolle der Entwicklung ermöglichte. Gegen Ende der 1920er Jahre kamen viele Kinder bereits mit einer „Untersuchungsakte“ zur ersten Schuluntersuchung, welche bereits in der Säuglingsfürsorgestelle angelegt worden war. ${ }^{24}$

Eine besonders wichtige Aufgabe der Schulärzte war die eingehende Untersuchung der Schulanfänger. Aus dem Bericht des Schulärztlichen Dienstes in Leoben für das Schuljahr 1921/22 geht hervor, dass im Rahmen von 100 Schulsprechstunden 685 Kinder erst- und 369 Kinder nachuntersucht wurden. 49 Kinder waren aufgrund von Tuberkuloseverdacht an die entsprechende Fürsorgestelle weiter verwiesen worden, und nicht weniger als 183 - also mehr als ein Viertel der Untersuchten! - wurden aufgrund verschiedener Erkrankungen zur Behandlung zu ihrem Hausarzt überwiesen. Sechs Kinder wurden wegen Schulunfähigkeit um ein Jahr zurückgestellt. Eine genauere Augenuntersuchung wurde bei 28 Kindern vorgenommen, von denen vier an die Grazer Augenklinik überstellt wurden. Da sie die anderen Schüler gefährdeten, wurden weiters

20 Obersteirische Volkszeitung, Nr. 25, 3.3.1925, S. 3, Leobner Zeitung Nr. 89, 4.8.1927, S. 1 - 2.

21 Obersteirische Volkszeitung, Nr. 54, 9.7.1904, S. 7.

22 Obersteirische Volkszeitung, Nr. 103, 31.12.1919, S. 3.

23 Obersteirische Volkszeitung, Nr. 98, 17.12.1977, S. 3.

24 Obersteirische Volkszeitung, Nr. 51, 5.5.1927, S. 4. 
25 Kinder mit ansteckenden Krankheiten für einige Zeit vom Unterricht suspendiert. Fünf Kinder wurden auf schulärztlichen Ratschlag hin operiert, und drei davon aufgrund der Dringlichkeit sofort in Krankenhaus gebracht. ${ }^{25}$

Die Schulärzte erledigten auch die schriftlichen Ansuchen um Aufnahme bei Heilstätten und anderen Kliniken. Weiters hielten sie Vorträge für interessierte Eltern beispielsweise über die Gefahren von Tuberkulose oder Rachitis. Außerdem führten die Schulärzte Impfungen gegen Pocken an den Schulen durch. Im Schuljahr 1921/22 waren es 228 Impflinge gewesen. ${ }^{26}$ Diese Statistik zeigt recht eindrucksvoll die vielfältigen Aufgaben der damaligen Schulärzte.

Während des Schuljahres 1923/24 wurden vom Leobner Schularzt Dr. Robert Tischitz sogar 1.234 Kinder untersucht, von denen allerdings nur 135 als gesund eingestuft wurden. Bei den übrigen wurden verschiedenste Probleme erkannt, die - von „oben nach unten" betrachtet - von Sehschwächen bis zu Plattfüßen reichten aber auch ernste Erkrankungen festgestellt. Unter den „ausgemusterten“ Kindern waren auch relativ viele vernachlässigte Kinder mit Floh-, Wanzen-, Wurm- oder Lausbefall. Ebenso genannt werden in dem betreffenden Zeitungsbericht Kinder mit Zahnkaries, Augenentzündungen, Abszessen, Nervenleiden oder Kropfbildung. Festgestellt wurden auch Brandwunden, Hundebisse und sogar ein Fall von schwerer Schwefelsäureverätzung. Am häufigsten - in 269 Fällen, also bei nahezu jedem 4. untersuchten Schulkind - traten aber Lungenkrankheiten in Erscheinung. Bei 23 Kindern wurde ein behandlungsbedürftiger Herzfehler festgestellt. Den jeweiligen Leiden entsprechend wurde Rücksprache mit den Eltern gehalten, nötigenfalls die Fürsorge eingeschaltet bzw. das Kind an einen Hausarzt, eine Heilstätte oder ein Krankenhaus überwiesen und die gesetzten Maßnahmen schließlich neuerlich vom Schularzt kontrolliert. Die Statistiken über die Schuluntersuchungen wurden auch dem Ortsschulrat vorgelegt. ${ }^{27}$

Die Zahnbehandlung der Schulkinder wurde dank eines Übereinkommens von den Krankenkassen übernommen; für jene Kinder, deren Eltern keine Krankenversicherung hatten, bezahlte die Gemeinde die Zahnbehandlungskosten. Vorbeugend wirkte die Unterweisung in richtigem Zähnputzen mehrmals jährlich an der Schule durch eigens geschulte Fürsorgeschwestern. Den Kindern wurde der richtige Umgang mit Zahnbürsten und Zahnpulver erklärt und die Wichtigkeit der Mundhygiene näher gebracht.

Leichteren Fällen von Haltungsschäden wurde durch spezielle Turnübungen, die in der Schule beigebracht und ausgeführt wurden, entgegen gewirkt. In Donawitz gab es weiters die Möglichkeit, ein „Schulbad“ aufzusuchen und in der „Entlausungsanlage“ lästige Parasiten los zu werden. ${ }^{28}$ Das Zimmer für die Schuluntersuchungen in Donawitz befand sich im ersten Stock der Mädchenschule. Der Schularzt wurde von zwei Fürsorgeschwestern unterstützt, die auch die Untersuchungsergebnisse in die Karteikarten eintrugen. ${ }^{29}$

Nach zehn Jahren in der Schulfürsorge zeichnete Dr. Jellenigg in einem Bericht an den Donawitzer Ortsschulrat ein sehr positives Bild über den langfristigen Erfolg der schulärztlichen Untersuchungen: Auf sozialem, hygienischem und medizinischem Ge-

25 Obersteirische Volkszeitung, Nr. 88, 5.8.1922, S. 4.

26 Obersteirische Volkszeitung, Nr. 88, 5.8.1922, S. 4.

27 Obersteirische Volkszeitung, Nr. 79, 15.7.1924, S. 3.

28 Obersteirische Volkszeitung, Nr. 53, 10.5.1927, S. 4.

29 Obersteirische Volkszeitung, Nr. 53, 10.5.1927, S. 4. 
biet ließen sich sicher Auffassung nach große Erfolge verzeichnen. Trotz der ökonomischen Notlage Mitte der 1930er Jahre war die Häufigkeit der Tuberkulose stark zurückgegangen, und Gelenks- oder Knochentuberkulose unter den Schulkindern war gänzlich verschwunden. Aufgrund der langfristigen Aufzeichnungen der Gesundheitsdaten konnte der Schularzt auch berufsberatend wirken und den Schulabgängern jene Berufssparten empfehlen, die für sie aus medizinischer Sicht am besten geeignet waren. Herausgestrichen wurden in diesem Bericht auch die hohen Kosteneinsparungen aufgrund wirkungsvoller Vorbeugemaßnahmen, die vor allem den Eltern und der öffentlichen Hand zugute kamen. Nicht unerwähnt blieb auch der Nebeneffekt, dass die Kinder durch die Schuluntersuchungen daran gewöhnt würden, im Bedarfsfall rechtzeitig einen Arzt zu Rate zu ziehen. ${ }^{30}$

Die soeben geschilderte Gesundheitsfürsorge des Bezirkes wurde während der Jahre des Ständestaates wie gewohnt weitergeführt. In den kleineren Ortschaften, wozu auch Göss zählte, bestand diese nunmehr als „Einheitsfürsorge“. Hier wurde Schwangern-, Säuglings-, Kleinkinder und Lungenfürsorge zusammen von einem Arzt und einer Fürsorgerin durchgeführt. In den größeren Orten wurden die einzelnen Fürsorgezweige auf jeweils spezialisierte Ärzte und Fürsorgerinnen aufgeteilt.

Insbesondere die städtische Säuglingsfürsorge- und Mütterberatungsstelle in Leoben, nunmehr unter der Leitung von Dr. Helmut Zeder, erfreute sich regen Zulaufs. Die Statistik zeigte nunmehr aber eine leichte Zunahme der Kindersterblichkeit sowie einen auffälligen Anstieg an Tuberkulosefällen, die vor allem auf die schlechte wirtschaftliche Lage zurückgeführt wurden. ${ }^{31}$

Ein Leobner Pressebericht aus dem Jahr 1937 thematisiert schließlich die Frage der Ausbildung der Ärzte in Bezug auf sozialärztliche Aufgaben, welche bislang ungenügend gewesen sei. Am 7. Oktober 1937 wurde daher in Graz ein kostenloser, dreitägiger Kurs abgehalten, der sich im Besonderen an Landärzte richtete. Den Ärzten wurden die Aufgaben in der Schwangeren-, Mütter-, Säuglings und Schulfürsorge näher gebracht. Ein Schwerpunkte lag dabei auf der Bekämpfung der Tuberkulose. Weitere Themen von Vorträgen waren: Armenarzt und Armenfürsorge, Kriegsbeschädigten- und Krüppelfürsorge, die Tätigkeit des Arztes und das Gesundheitswesen in der Sozialversicherung, Grundlagen der Seuchenbekämpfung und Gerichtsärztliche Befundtechniken sowie Erstversorgung von "Geisteskranken" und sozialhygienische Volkserziehung. ${ }^{32}$

Nach der Machtübernahme der Nationalsozialisten übernahm das Leobner Gesundheitsamt einen Teil der Fürsorgeaufgaben. Als Leiter fungierte Medizinalrat Dr. Günther Ofner. ${ }^{33}$ Auf die weitere Entwicklung während des Zweiten Weltkriegs sowie nach 1945 soll aber an anderer Stelle ausführlicher eingegangen werden.

30 Obersteirische Volkszeitung, Nr. 51, 2.5.1935, S. 4.

31 Obersteirische Volkszeitung, Nr. 71. 20.6.1936, S. 6.

32 Obersteirische Volkszeitung, Nr. 109, 21.9.1937, S. 5. Zehn finanziell schwächer gestellten Ärzten, vorzüglich Distriktsärzten, wurde hierbei übrigens eine Fahrtkostenbeihilfe von 30 Schilling zur Teilnahme an der Veranstaltung gewährt.

33 Obersteirische Volkszeitung, Nr. 148, 15.12.1942, S. 3. 